\title{
HAT-P-16b: A $4 M_{\mathrm{J}}$ PLANET TRANSITING A BRIGHT STAR ON AN ECCENTRIC ORBIT* ${ }^{\dagger}$
}

\author{
L. A. Buchhave ${ }^{1,2}$, G. Á. Bakos ${ }^{1,9}$, J. D. Hartman ${ }^{1}$, G. Torres ${ }^{1}$, G. Kovács ${ }^{3}$, D. W. Latham ${ }^{1}$, R. W. Noyes $^{1}$, \\ G. A. Esquerdo ${ }^{1}$, M. Everett ${ }^{1}$, A. W. Howard ${ }^{4}$, G. W. Marcy ${ }^{4}$, D. A. Fischer ${ }^{5}$, J. A. Johnson ${ }^{6}$, J. Andersen $^{2,7}$, G. Fürész $^{1}$, \\ G. Perumpilly ${ }^{1}$, D. D. Sasselov ${ }^{1}$, R. P. Stefanik ${ }^{1}$, B. Béky ${ }^{1}$, J. LÁzÁR ${ }^{8}$, I. PAPP ${ }^{8}$, And P. SÁri ${ }^{8}$ \\ ${ }^{1}$ Harvard-Smithsonian Center for Astrophysics, Cambridge, MA 02138, USA \\ ${ }^{2}$ Niels Bohr Institute, Copenhagen University, Denmark \\ ${ }^{3}$ Konkoly Observatory, Budapest, Hungary \\ ${ }^{4}$ Department of Astronomy, University of California, Berkeley, CA 94720, USA \\ ${ }^{5}$ Department of Astronomy, Yale University, New Haven, CT 06511, USA \\ ${ }^{6}$ Department of Astrophysics, California Institute of Technology, MC 249-17, Pasadena, CA 91125, USA \\ ${ }^{7}$ Nordic Optical Telescope Scientific Association, La Palma, Canary Islands, Spain \\ ${ }^{8}$ Hungarian Astronomical Association, Budapest, Hungary \\ Received 2010 April 14; accepted 2010 July 5; published 2010 August 17
}

\begin{abstract}
We report the discovery of HAT-P-16b, a transiting extrasolar planet orbiting the $V=10.8$ mag F8 dwarf GSC 279201700 , with a period $P=2.775960 \pm 0.000003$ days, transit epoch $T_{c}=2455027.59293 \pm 0.00031\left(\mathrm{BJD}^{10}\right)$, and transit duration $0.1276 \pm 0.0013$ days. The host star has a mass of $1.22 \pm 0.04 M_{\odot}$, radius of $1.24 \pm 0.05 R_{\odot}$, effective temperature $6158 \pm 80 \mathrm{~K}$, and metallicity $[\mathrm{Fe} / \mathrm{H}]=+0.17 \pm 0.08$. The planetary companion has a mass of $4.193 \pm 0.094 M_{\mathrm{J}}$ and radius of $1.289 \pm 0.066 R_{\mathrm{J}}$, yielding a mean density of $2.42 \pm 0.35 \mathrm{~g} \mathrm{~cm}^{-3}$. Comparing these observed characteristics with recent theoretical models, we find that HAT-P-16b is consistent with a 1 Gyr $\mathrm{H} / \mathrm{He}$-dominated gas giant planet. HAT-P-16b resides in a sparsely populated region of the mass-radius diagram and has a non-zero eccentricity of $e=0.036$ with a significance of $10 \sigma$.
\end{abstract}

Key words: planetary systems - stars: individual (HAT-P-16, GSC 2792-01700) - techniques: photometric techniques: spectroscopic

Online-only material: color figure, machine-readable table

\section{INTRODUCTION}

Planets that transit their host stars play a special role in our understanding of the characteristics of exoplanets: their transit allows us to accurately determine the radius and the orbital inclination of the planet from the light curve so that the ambiguity due to orbital inclination in the planetary mass as derived from the spectroscopic orbit of the host star can be largely eliminated. The mass and radius enable us to infer a bulk composition of the planet, and although there are degeneracies associated with the bulk composition, it allows us to put constraints on models of planetary structure and formation theories. The incredible diversity of the over 80 discovered transiting planets, ranging from dense planets with a higher mean density than that of copper to strongly irradiated puffed-up planets with a mean density comparable to that of corkwood, has baffled the exoplanet community, and no unified theory has been established to explain all the systems consistently. Transiting extrasolar planet (TEP) discoveries are primarily the result of dedicated ground-based searches, such as SuperWASP (Pollacco et al. 2006), the Hungarian-made Automated Telescope Network (HATNet; Bakos et al. 2004), TrES (Alonso et al. 2004), and XO (McCullough et al. 2005),

\footnotetext{
* Based in part on observations made with the Nordic Optical Telescope, operated on the island of La Palma jointly by Denmark, Finland, Iceland, Norway, and Sweden, in the Spanish Observatorio del Roque de los Muchachos of the Instituto de Astrofisica de Canarias.

† Based in part on observations obtained at the W. M. Keck Observatory, which is operated by the University of California and the California Institute of Technology. Keck time has been granted by NASA (N018Hr).

9 NSF Fellow.

${ }^{10}$ All BJD values given in this paper are UTC based (e.g., Torres et al. 2010).
}

and space-borne searches, such as CoRoT (Auvergne et al. 2009) and Kepler (Borucki et al. 2010).

Since its commissioning in 2003, the HATNet (Bakos et al. 2004) survey has been one of the major contributors to the discoveries of TEPs. HATNet has discovered over a dozen TEPs since 2006 by surveying bright stars ( $8 \mathrm{mag} \lesssim I \lesssim 12.5 \mathrm{mag}$ ) in the Northern hemisphere and has now covered approximately $11 \%$ of the Northern sky. HATNet consists of six wide field automated telescopes: four of these are located at the Fred Lawrence Whipple Observatory (FLWO) in Arizona and two on the roof of the Submillimeter Array hangar of the Smithsonian Astrophysical Observatory (SAO) in Hawaii. In this paper, we report a new TEP discovery of HATNet, called HAT-P-16b.

The structure of the paper is as follows. In Section 2, we summarize the observations, including the photometric detection, and follow-up observations. In Section 3, we describe the analysis of the data, such as the stellar parameter determination (Section 3.1), ruling out blend scenarios (Section 3.2), and global modeling of the data (Section 3.3). We discuss our findings in Section 4.

\section{OBSERVATIONS}

\subsection{Photometric Detection}

The transits of HAT-P-16b were detected with the HAT- 6 and HAT-7 telescopes in Arizona and the HAT- 8 and HAT-9 telescopes in Hawaii. The star GSC 2792-01700 (also known as 2MASS $00381756+4227470 ; \alpha=00^{\mathrm{h}} 38^{\mathrm{m}} 17.56, \delta=$ $+42^{\circ} 27^{\prime} 47^{\prime \prime}$. 1 ; J2000; $V=10.812$; Droege et al. 2006) lies in the intersection of three separate HATNet fields internally labeled as 123,163 , and 164 . Field 123 was observed with an $I$ filter and a $2 \mathrm{~K} \times 2 \mathrm{~K} \mathrm{CCD}$, while fields 163 and 164 
were observed through $R$ filters with $4 \mathrm{~K} \times 4 \mathrm{~K}$ CCDs. All three fields were observed with a 5 minute exposure time and at a 5.5 minute cadence in the period between 2007 July and 2008 September during which over 12,000 exposures were gathered for the three fields. Each image contains between 27,000 and 76,000 stars down to a magnitude of $I \sim 13$ for field 123 and $R \sim 15$ for fields 163 and 164, yielding a photometric precision for the brightest stars in the field of $\sim 2$ mmag for field 123 and $\sim 5$ mmag for fields 163 and 164 .

Frame calibration, astrometry, and aperture photometry were done in an identical way to recent HATNet TEP discoveries, as described in Bakos et al. (2010) and Pál (2009). The resulting light curves were decorrelated (cleaned of trends) using the External Parameter Decorrelation (EPD) technique in "constant" mode (see Bakos et al. 2010) and the Trend Filtering Algorithm (TFA; see Kovács et al. 2005). The light curves were searched for periodic box-like signals using the Box Least-Squares method (BLS; see Kovács et al. 2002). A significant signal was detected in the light curve of GSC 279201700 , with a depth of $\sim 10.2 \mathrm{mmag}$, and a period of $P=$ 2.7760 days. The dip had a relative duration (first to last contact) of $q \approx 0.0460 \pm 0.0005$, corresponding to a total duration of $P q \approx 3.062 \pm 0.031 \mathrm{hr}$ (see Figure 1$)$.

\subsection{Reconnaissance Spectroscopy}

Transiting planet candidates found by ground-based, widefield photometric surveys must undergo a rigorous vetting process to eliminate the many astrophysical systems mimicking transiting planets (called false positives), the rate of which has proved to be much higher than the occurrence of true planets (10-20 times higher). Low signal-to-noise ratio $(\mathrm{S} / \mathrm{N})$ highresolution reconnaissance spectra are used to extract stellar parameters such as effective temperature, gravity, metallicity, and rotational and radial velocities (RVs) to rule out these false positives. Examples of false positives which are discarded are eclipsing binaries and triple systems. The latter can be either hierarchical or chance alignment systems where the light of the eclipsing pair of stars is diluted by the light of a third brighter star. Rapidly rotating and/or hot host stars whose spectrum is unsuitable for high-precision velocity work are also discarded.

We acquired seven reconnaissance spectra with the CfA Digital Speedometer (DS; Latham 1992) mounted on the FLWO $1.5 \mathrm{~m}$ Tillinghast Reflector between 2008 December and 2009 January. The extracted modest precision RVs gave a mean absolute RV $=-16.83 \mathrm{~km} \mathrm{~s}^{-1}$ with an rms of $0.51 \mathrm{~km} \mathrm{~s}^{-1}$, which is consistent with no detectable RV variation. The stellar parameters derived from the spectra (Torres et al. 2002), including the effective temperature $T_{\mathrm{eff} \star}=6000 \pm 100 \mathrm{~K}$, surface gravity $\log g_{\star}=4.0 \pm 0.25(\log \mathrm{cgs})$ and projected rotational velocity $v \sin i=3.8 \pm 1.0 \mathrm{~km} \mathrm{~s}^{-1}$, correspond to an F8 dwarf.

\subsection{High-resolution, High S/N Spectroscopy}

The high significance of the transit detection by HATNet, together with the stellar spectral type and small RV variations, encouraged us to gather high-resolution, high $\mathrm{S} / \mathrm{N}$ spectra to determine the orbit of the system. We have taken 21 spectra between 2009 August and October using the FIbre-fed Échelle Spectrograph (FIES) at the $2.5 \mathrm{~m}$ Nordic Optical Telescope (NOT) at La Palma, Spain (Djupvik \& Andersen 2009). We used the medium- and the high-resolution fibers ( 1 ". 3 projected diameter) with resolving powers of $\lambda / \Delta \lambda \approx 46,000$ and 67,000 , re-
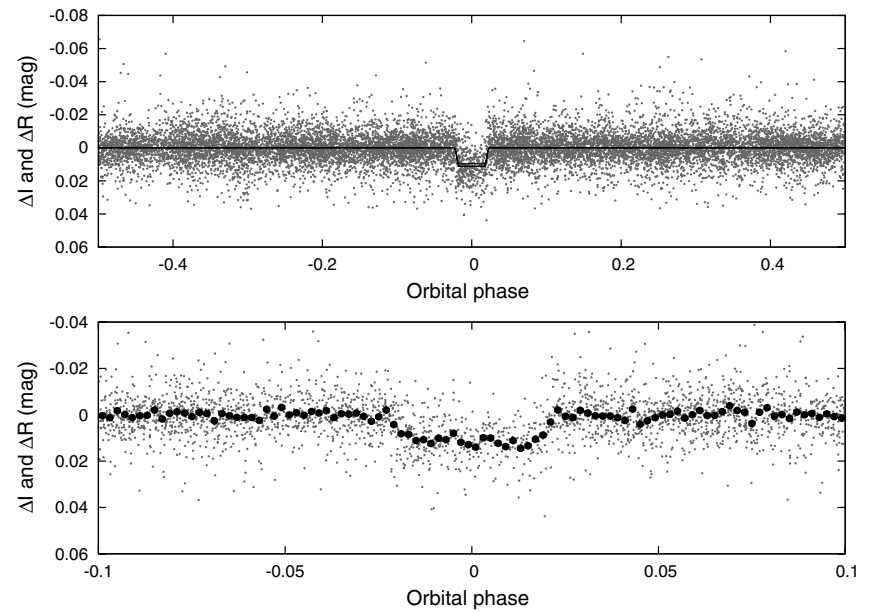

Figure 1. Unbinned light curve of HAT-P-16 including all 12,552 instrumental $I$ band and $R$ band 5.5 minute cadence measurements obtained with the HAT-6, HAT-7, HAT-8, and HAT-9 telescopes (see the text for details), and folded with the period of $P=2.7759602$ days (which is the result of the fit described in Section 3 ). The $I$-band and $R$-band data are merged in this figure. The solid line shows the transit model fit to the light curve (Section 3.3). In the lower panel, the large solid circles represent the binned average of the photometric measurements with a bin size of 0.005 days.

spectively, giving a wavelength coverage of $\sim 3600-7400 \AA$. We used the wavelength range from approximately $\sim 4000-5500 \AA$ to determine the RVs. The exposure time was approximately 20 minutes yielding an $\mathrm{S} / \mathrm{N}$ from 45 to $85 \mathrm{pixel}^{-1}$ in the wavelength range used.

We also used the High-Resolution Echelle Spectrometer (HIRES) instrument (Vogt et al. 1994) on the Keck I telescope located on Mauna Kea, Hawaii. We obtained six exposures with an iodine cell, plus one iodine-free template with Keck I/HIRES. The S/N per pixel ranged from 90 to 120 and the exposure time was approximately 10 minutes. The observations were made on six nights between 2009 July 3 and 2009 October 29. The width of the spectrometer slit used on HIRES was 0 .'86, resulting in a resolving power of $\lambda / \Delta \lambda \approx 55,000$, with a wavelength coverage of $\sim 3800-8000 \AA$. The iodine gas absorption cell was used to superimpose a dense forest of $\mathrm{I}_{2}$ lines on the stellar spectrum and establish an accurate wavelength fiducial (see Marcy \& Butler 1992) and only the part of the spectrum where the iodine lines are present was used in the determination of the RVs. Relative RVs in the solar system barycentric frame were derived as described by Butler et al. (1996), incorporating full modeling of the spatial and temporal variations of the instrumental profile.

The final RV data and their errors (for both instruments) are listed in Table 1. The folded data are plotted in Figure 2. The systemic gamma velocities (that are the result of the global modeling, as laid out in Section 3) have been subtracted to ensure a common zero point. The best orbital fit (see Section 3) is superimposed in the figure.

In the same figure, we also show the relative $S$ index, which is a measure of the chromospheric activity of the star derived from the flux in the cores of the $\mathrm{Ca}$ II $\mathrm{H}$ and $\mathrm{K}$ lines. This index was computed following the prescription given by Vaughan et al. (1978), after matching each spectrum to a reference spectrum using a transformation that includes a wavelength shift and a flux scaling that is a polynomial as a function of wavelength. The transformation was determined on the regions of the spectra that are not used in computing this indicator. We also measured the $R_{H K}^{\prime}$ index for the system to be $R_{H K}^{\prime}=-4.862$, as described 
Table 1

Relative Radial Velocity and Bisector Span Variation Measurements of HAT-P-16

\begin{tabular}{|c|c|c|c|c|c|}
\hline $\begin{array}{c}\text { BJD } \\
(2,455,000+)\end{array}$ & $\begin{array}{c}\mathrm{RV}^{\mathrm{a}} \\
\left(\mathrm{m} \mathrm{s}^{-1}\right)\end{array}$ & $\begin{array}{c}\sigma_{\mathrm{RV}} \\
\left(\mathrm{m} \mathrm{s}^{-1}\right)\end{array}$ & $\begin{array}{c}\mathrm{BS} \\
\left(\mathrm{m} \mathrm{s}^{-1}\right)\end{array}$ & $\begin{array}{c}\sigma_{\mathrm{BS}} \\
\left(\mathrm{m} \mathrm{s}^{-1}\right)\end{array}$ & Inst. \\
\hline 048.64476 & -137.7 & 4.6 & 16.2 & 11.9 & FIES h \\
\hline $049.70638 \ldots \ldots \ldots$ & -339.5 & 10.5 & -4.8 & 16.5 & FIES h \\
\hline $050.68239 \ldots \ldots \ldots$ & -911.9 & 6.1 & -21.3 & 12.5 & FIES h \\
\hline $051.73142 \ldots \ldots \ldots$ & 60.3 & 5.8 & 3.5 & 11.1 & FIES h \\
\hline $052.68956 \ldots \ldots \ldots$ & -579.8 & 4.4 & -6.0 & 9.9 & FIES h \\
\hline $053.71751 \ldots \ldots \ldots$ & -674.7 & 11.3 & 8.8 & 20.5 & FIES h \\
\hline $054.71713 \ldots \ldots \ldots$ & 66.2 & 5.7 & 3.6 & 9.2 & FIES h \\
\hline $107.58955 \ldots \ldots \ldots$ & 0.7 & 5.2 & 6.0 & 7.6 & FIES m \\
\hline $108.62791 \ldots \ldots \ldots$ & -965.2 & 5.3 & -4.6 & 7.2 & FIES m \\
\hline $109.56094 \ldots \ldots \ldots$ & -310.4 & 7.0 & 0.9 & 12.5 & FIES m \\
\hline $110.54715 \ldots \ldots \ldots$ & -128.9 & 7.5 & -12.1 & 6.4 & FIES m \\
\hline $111.56509 \ldots \ldots \ldots$ & -989.4 & 4.1 & 2.5 & 5.0 & FIES m \\
\hline $112.60304 \ldots \ldots \ldots$ & -53.7 & 5.5 & 0.3 & 8.4 & FIES m \\
\hline $113.50725 \ldots \ldots \ldots$ & -294.7 & 4.7 & 14.9 & 6.4 & FIES m \\
\hline $114.53303 \ldots \ldots \ldots$ & -924.1 & 4.5 & -1.5 & 7.7 & FIES m \\
\hline $116.52227 \ldots \ldots \ldots$ & -558.0 & 5.3 & -1.3 & 8.0 & FIES m \\
\hline $122.51752 \ldots \ldots \ldots$ & -941.1 & 7.5 & -0.6 & 10.2 & FIES $m$ \\
\hline $123.46870 \ldots \ldots \ldots$ & -262.4 & 4.7 & 1.5 & 8.4 & FIES m \\
\hline $124.48749 \ldots \ldots \ldots$ & -161.9 & 7.9 & 0.9 & 12.0 & FIES m \\
\hline $125.44928 \ldots \ldots \ldots$ & 1008.8 & 5.9 & -8.1 & 9.0 & FIES m \\
\hline $126.47958 \ldots \ldots \ldots$ & -29.8 & 5.2 & 1.3 & 8.2 & FIES m \\
\hline $017.09285 \ldots \ldots \ldots$ & -531.7 & 2.3 & -3.1 & 4.3 & HIRES \\
\hline $019.09413 \ldots \ldots \ldots$ & 179.5 & 2.6 & -18.1 & 5.8 & HIRES \\
\hline $107.08869 \ldots \ldots \ldots$ & $\ldots$ & $\ldots$ & -10.3 & 4.6 & HIRES \\
\hline $107.09645 \ldots \ldots \ldots$ & 445.3 & 2.4 & -2.9 & 3.9 & HIRES \\
\hline $108.97959 \ldots \ldots \ldots$ & -497.6 & 2.6 & 33.5 & 1.7 & HIRES \\
\hline $112.12355 \ldots \ldots \ldots$ & -109.3 & 2.8 & 6.7 & 2.1 & HIRES \\
\hline $135.02181 \ldots \ldots \ldots$ & 510.9 & 2.5 & -5.8 & 4.6 & HIRES \\
\hline
\end{tabular}

Notes. For the iodine-free template exposure, we do not measure the RV but do measure the BS. Such template exposures can be distinguished by the missing RV value. The "Inst." column refers to the instrument used, i.e., the FIES spectrograph at the Nordic Optical Telescope (NOT) using the medium- and high-resolution fibers or the HIRES spectrograph at Keck I. $\sigma_{\mathrm{RV}}$ and $\sigma_{\mathrm{BS}}$ are formal statistical errors.

${ }^{a}$ The fitted zero point that is on an arbitrary scale (denoted as $\gamma_{\text {rel }}$ in Section 3.3) has not been subtracted from the velocities.

by Noyes et al. (1984). Note that our relative $S$ index has not been calibrated to the scale of Vaughan et al. (1978). We do not detect any significant variation of the index correlated with orbital phase; such a correlation might have indicated that the RV variations could be due to stellar activity, casting doubt on the planetary nature of the candidate.

Since this is the first time the NOT has been used to determine the orbit of a HATNet planet, we describe briefly the extraction of the RVs. We have built a custom pipeline to rectify and crosscorrelate spectra from Échelle spectrographs with the goal of providing very precise RVs. The first step in the extraction process is to remove the bias level and crop the raw images. To effectively remove cosmic rays, the observation is divided into three separate exposures, which enables us to combine the raw images using median filtering, removing virtually all cosmic rays. We use a flat field to trace the Échelle orders and we rectify the spectra by using the "optimal extraction" algorithm (Hewett et al. 1985; Horne 1986). The blaze function is determined by fitting cubic splines to a combined high $\mathrm{S} / \mathrm{N}$ flat field exposure and is saved separately in order to preserve the original flux in the stellar exposure. By dividing the normalized blaze function into the rectified flat field spectrum, we can determine the pixel to pixel variations and correct for these. The scattered light in
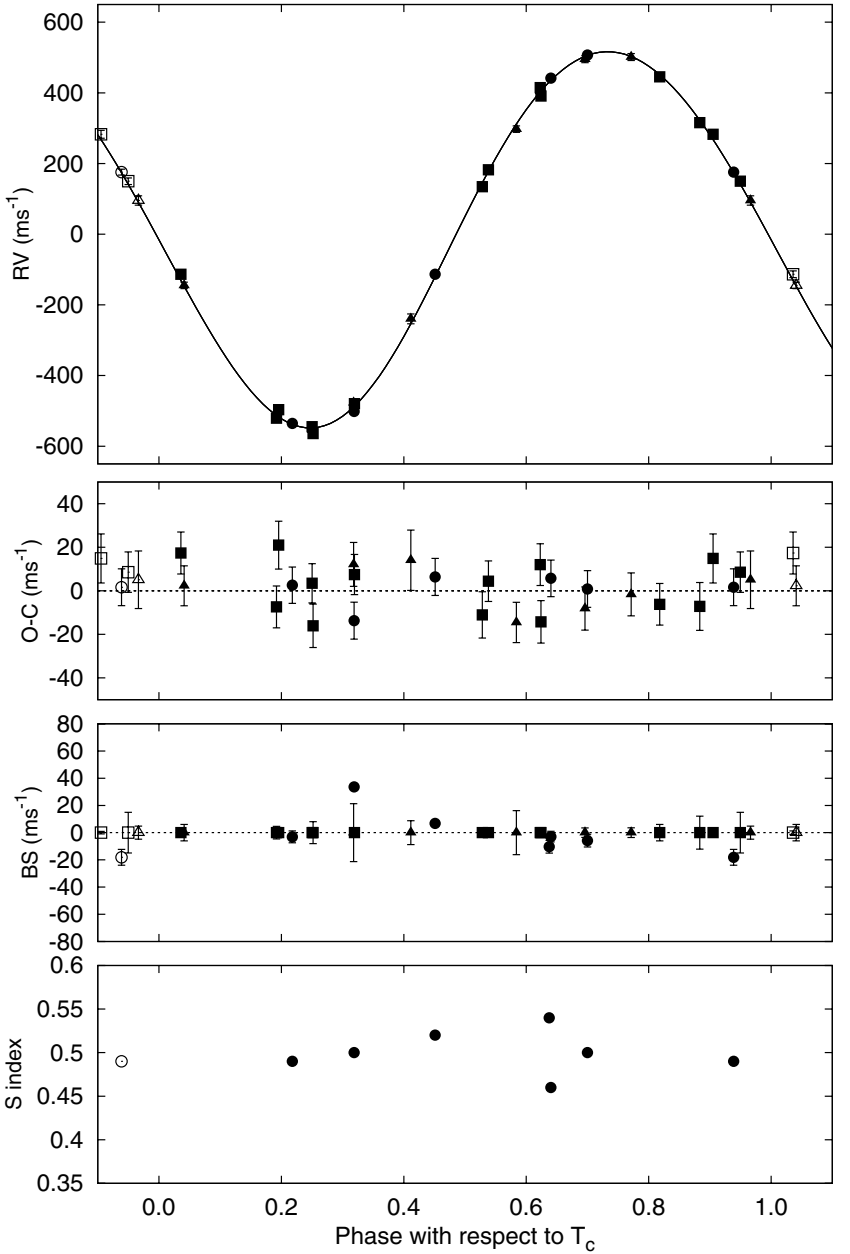

Figure 2. Top: RV measurements from the Nordic Optical Telescope (NOT) and Keck for HAT-P-16, along with an orbital fit, shown as a function of orbital phase, using our best-fit period (see Section 3). Velocities from NOT using the mediumand high-resolution fiber are shown as squares and triangles, respectively, and the Keck velocities are shown as circles. Zero phase is defined by the transit midpoint. The center-of-mass velocity has been subtracted. Note that the error bars include the stellar jitter, added in quadrature to the formal errors given by the spectrum reduction pipeline. Second panel: phased residuals after subtracting the orbital fit (also see Section 3). The rms variation of the residuals is about $10.0 \mathrm{~m} \mathrm{~s}^{-1}$. Third panel: bisector span (BS) variations including the template spectrum (Section 3.2). Bottom: relative $S$ activity index values which have only been calculated for the Keck spectra. Our relative $S$ index has not been calibrated to the scale of Vaughan et al. (1978). Note the different vertical scales of the panels.

the two-dimensional raw image is determined and removed by masking out the signal in the Échelle orders and fitting the interorder scattered light flux with a two-dimensional polynomial.

Thorium argon (ThAr) calibration images are obtained through the science fiber before and after the stellar observation. The two calibration images are combined to form the basis for the fiducial wavelength calibration. We have determined that the best wavelength solution is achieved by choosing an exposure time that saturates the strong argon lines but enhances the forest of weaker thorium lines.

FIES is not a vacuum spectrograph and is only temperature controlled to $0.1{ }^{\circ} \mathrm{C}$. Consequently, the $\mathrm{RV}$ errors are dominated by our limited ability to correct for shifts due to pressure, humidity, and temperature variations. In order to successfully remove these large variations $\left(>1.5 \mathrm{~km} \mathrm{~s}^{-1}\right)$, it is critical that the ThAr light travels through the same light path as the stellar light and thus acts as an effective proxy to remove these variations. 


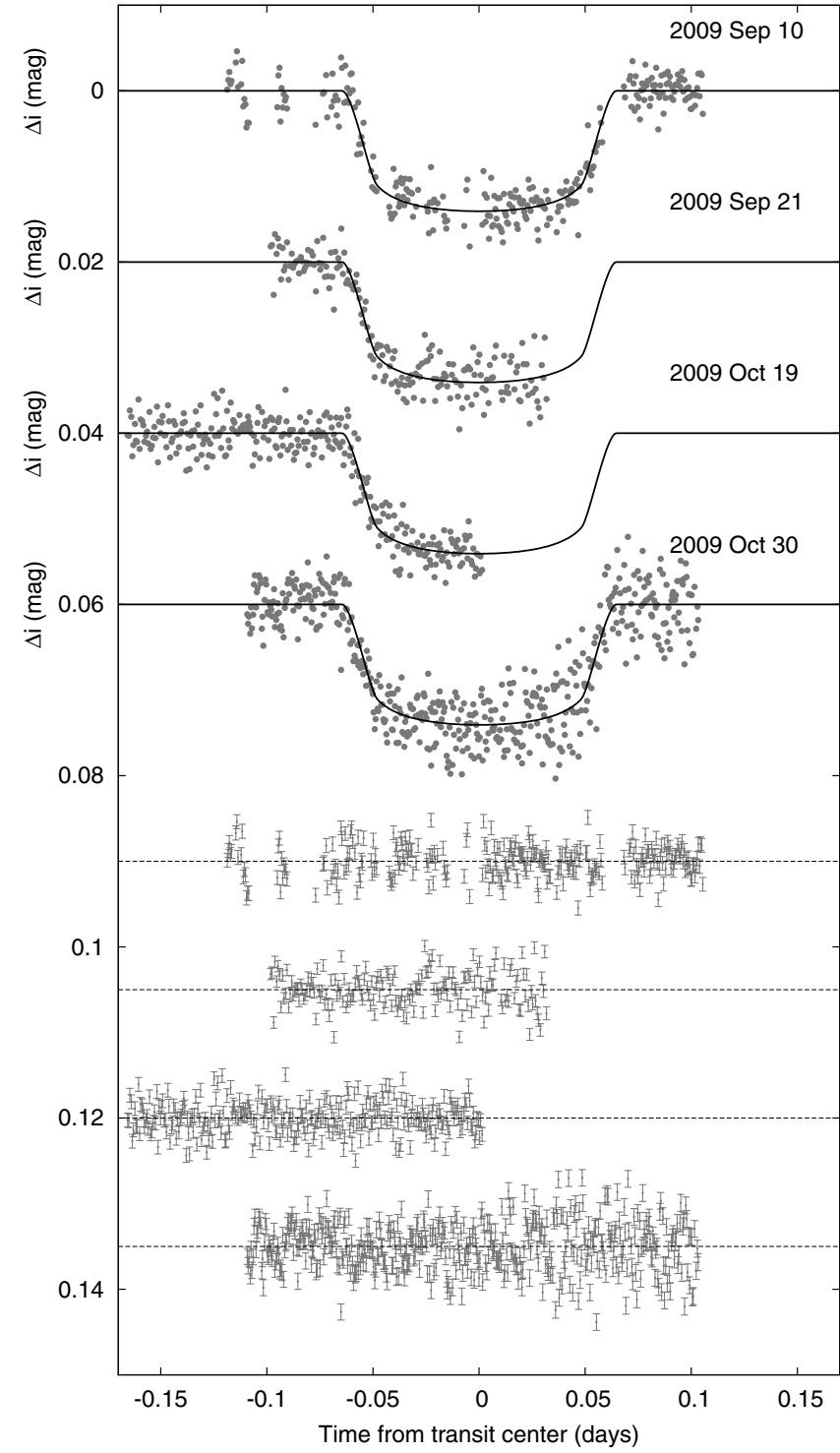

Figure 3. Unbinned instrumental $i$-band transit light curves, acquired with KeplerCam at the FLWO $1.2 \mathrm{~m}$ telescope. Superimposed are the best-fit transit model light curves. At the bottom of the figure, we show the residuals from the fit. Error bars represent the photon and background shot noise, plus the readout noise.

We have therefore chosen to interleave the stellar observation between two ThAr exposures instead of using the simultaneous ThAr technique, which may not exactly describe the induced shifts in the science fiber. The centers of the ThAr lines are found by fitting a Gaussian function to the line profiles and a two-dimensional fifth-order Legendre polynomial is used to describe the wavelength solution.

Once the spectra have been extracted, a multi-order crosscorrelation is performed order by order. First, the spectra are interpolated to a common oversampled log wavelength scale with the same monotonic wavelength increment in all orders. A high and low pass filter is applied in the Fourier domain and the ends of the spectra are apodized with a cosine bell function. The orders are cross-correlated using a fast Fourier transform and the cross-correlation function (CCF) for each order is coadded. This automatically weights each order by its flux, giving more weight to the orders with more photons. This CCF peak is fitted with a Gaussian function to determine its center. The internal precision is estimated by finding the RV for each order
Table 2

Follow-up Photometry of HAT-P-16

\begin{tabular}{crccc}
\hline \hline $\begin{array}{c}\text { BJD } \\
(2,400,000+)\end{array}$ & \multicolumn{1}{c}{ Mag $^{\mathrm{a}}$} & $\sigma_{\text {Mag }}$ & Mag(orig) & Filter \\
\hline 55085.69759 & 0.00368 & 0.00082 & 9.93719 & $i$ \\
55085.69808 & 0.00111 & 0.00082 & 9.93426 & $i$ \\
55085.69860 & -0.00132 & 0.00082 & 9.93260 & $i$ \\
55085.69911 & -0.00200 & 0.00082 & 9.93017 & $i$ \\
55085.69962 & 0.00022 & 0.00082 & 9.93280 & $i$ \\
\hline
\end{tabular}

Notes.

a The out-of-transit level has been subtracted. These magnitudes have been subjected to the EPD and TFA procedures, carried out simultaneously with the transit fit.

b These are raw magnitude values without application of the EPD and TFA procedures.

(This table is available in its entirety in a machine-readable form in the online journal. A portion is shown here for guidance regarding its form and content.)

and the RV error is thus $\sigma=\operatorname{rms}(v) / \sqrt{N}$, where $v$ is the RV of the individual orders and $N$ is the number of orders. For the final RVs, a template spectrum is constructed by shifting and co-adding all the observed spectra, and the individual spectra are cross-correlated against this co-added template spectrum to minimize the contribution of noise in the template. We note that FIES has also been used to obtain a spectroscopic orbit for one of the first Kepler planets, namely Kepler- $7 b$ (Latham et al. 2010).

The observations were gathered while the moon was up, but the moon was well separated from the target on the sky and we had mostly clear conditions. Furthermore, the relative RV of the moon was well separated from the target, so if any lunar contamination did occur, it would have a negligible effect on the RVs.

\subsection{Photometric Follow-up Observations}

To confirm the transit signal and obtain high-precision light curves for modeling the system, we conducted photometric follow-up observations with the KeplerCam CCD on the FLWO $1.2 \mathrm{~m}$ telescope. We observed four transit events of HAT-P16 on the nights of 2009 September 10 and 21, October 19 and 30 (Figure 3). On the four nights, 303, 181, 293, and 471 frames were acquired with a cadence of $41,52,41$, and $32 \mathrm{~s}$ $(30,40,30$, and $20 \mathrm{~s}$ of exposure time) in the Sloan $i$ band, respectively.

The reduction of the images, including frame calibration, astrometry, and photometry, was performed as described in Bakos et al. (2010). We also performed EPD and TFA to remove trends simultaneously with the light curve modeling (for more details, see Section 3). The final light curves are shown in the upper plots of Figure 3, superimposed with our best-fit transit light curve models (see also Section 3); the photometry is provided in Table 2 .

\section{ANALYSIS}

\subsection{Properties of the Parent Star}

The stellar atmospheric parameters were derived from the template spectrum obtained with the Keck/HIRES instrument. We used the Spectroscopy Made Easy (SME) analysis package of Valenti \& Piskunov (1996), along with the atomic-line database of Valenti \& Fischer (2005). This yielded the following initial values and uncertainties (which we have conservatively increased to include our estimates of the systematic errors): 
Table 3

Stellar Parameters for HAT-P-16

\begin{tabular}{lcc}
\hline \hline \multicolumn{1}{c}{ Parameter } & Value & Source \\
\hline$T_{\mathrm{eff} \star}(\mathrm{K}) \ldots \ldots \ldots$ & $6158 \pm 80$ & $\mathrm{SME}^{\mathrm{a}}$ \\
{$[\mathrm{Fe} / \mathrm{H}](\mathrm{dex}) \ldots \ldots \ldots$} & $+0.17 \pm 0.08$ & $\mathrm{SME}$ \\
$v \sin i\left(\mathrm{~km} \mathrm{~s}^{-1}\right) \ldots$ & $3.5 \pm 0.5$ & $\mathrm{SME}$ \\
$v_{\mathrm{mac}}\left(\mathrm{km} \mathrm{s}^{-1}\right) \ldots$ & 4.61 & $\mathrm{SME}$ \\
$v_{\text {mic }}\left(\mathrm{km} \mathrm{s}^{-1}\right) \ldots$ & 0.85 & $\mathrm{SME}$ \\
$\gamma_{\mathrm{RV}}\left(\mathrm{km} \mathrm{s}^{-1}\right) \ldots$ & $-16.83 \pm 0.19$ & $\mathrm{DS}$ \\
$a_{i} \ldots \ldots \ldots$ & 0.2166 & $\mathrm{SME}+\mathrm{Claret}$ \\
$b_{i} \ldots \ldots \ldots$ & 0.3617 & $\mathrm{SME}+\mathrm{Claret}^{\mathrm{b}}$ \\
$M_{\star}\left(M_{\odot}\right) \ldots \ldots \ldots$ & $1.218 \pm 0.039$ & $\mathrm{YY}+a / R_{\star}+\mathrm{SME}^{\mathrm{c}}$ \\
$R_{\star}\left(R_{\odot}\right) \ldots \ldots \ldots$ & $1.237 \pm 0.054$ & $\mathrm{YY}+a / R_{\star}+\mathrm{SME}$ \\
$\log g_{\star}(\mathrm{cgs}) \ldots \ldots$ & $4.34 \pm 0.03$ & $\mathrm{YY}+a / R_{\star}+\mathrm{SME}$ \\
$L_{\star}\left(L_{\odot}\right) \ldots \ldots \ldots$ & $1.97 \pm 0.22$ & $\mathrm{YY}+a / R_{\star}+\mathrm{SME}$ \\
$v(\mathrm{mag}) \ldots \ldots \ldots$ & 10.812 & $\mathrm{TASS}$ \\
$M_{V}(\mathrm{mag}) \ldots \ldots \ldots$ & $4.03 \pm 0.13$ & $\mathrm{YY}+a / R_{\star}+\mathrm{SME}$ \\
$K(\mathrm{mag}, \mathrm{ESO})$ & $9.596 \pm 0.021$ & $2 \mathrm{MASS}+\mathrm{carpenter}^{\mathrm{d}}$ \\
$M_{K}(\mathrm{mag}, \mathrm{ESO})$ & $2.74 \pm 0.10$ & $\mathrm{YY}+a / R_{\star}+\mathrm{SME}$ \\
Age $(\mathrm{Gyr}) \ldots \ldots \ldots$ & $2.0 \pm 0.8$ & $\mathrm{YY}+a / R_{\star}+\mathrm{SME}$ \\
Distance $(\mathrm{pc}) \ldots \ldots \ldots$ & $235 \pm 10$ & $\mathrm{YY}+a / R_{\star}+\mathrm{SME}$ \\
\hline
\end{tabular}

\section{Notes.}

a $\mathrm{SME}=$ "Spectroscopy Made Easy" package for analysis of high-resolution spectra (Valenti \& Piskunov 1996). These parameters primarily depend on SME, with a small dependence on the iterative analysis incorporating the isochrone search and global modeling of the data, as described in the text.

b $\mathrm{SME}+$ Claret $=$ Based on the SME analysis and tables of quadratic limb-darkening coefficients from Claret (2004).

c $\mathrm{YY}+a / R_{\star}+\mathrm{SME}=\mathrm{YY} 2$ isochrones (Yi et al. 2001), $a / R_{\star}$ luminosity indicator, and SME results.

${ }^{\mathrm{d}}$ Based on the relations from Carpenter (2001).

effective temperature $T_{\text {eff }}=6175 \pm 80 \mathrm{~K}$, stellar surface gravity $\log g_{\star}=4.44 \pm 0.10$ (cgs), metallicity $[\mathrm{Fe} / \mathrm{H}]=$ $0.15 \pm 0.06 \mathrm{dex}$, and projected rotational velocity $v \sin i=$ $4.4 \pm 0.5 \mathrm{~km} \mathrm{~s}^{-1}$.

We could now use the effective temperature and the surface gravity found by SME to determine other stellar parameters, such as the mass, $M_{\star}$, and radius, $R_{\star}$, using model isochrones. However, there may be strong correlations between effective temperature, gravity, and metallicity in the spectroscopic determination of these parameters. Also, the effect of $\log g_{\star}$ on the spectral line shapes is typically subtle, and as a result $\log g_{\star}$ is generally a rather poor luminosity indicator. Instead, we used the $a / R_{\star}$, the normalized semimajor axis (related to $\rho_{\star}$, the mean stellar density), which can be derived directly from the light curves (Sozzetti et al. 2007).

The stellar parameters were determined simultaneously with the modeling of the light curves and RVs, as described next. We began by adopting the values of $T_{\mathrm{eff} \star},[\mathrm{Fe} / \mathrm{H}]$, and $\log g_{\star}$ from the SME analysis to fix the quadratic limb-darkening coefficients from the tabulations by Claret (2004), which are needed to model the light curves $\left(a_{i}, b_{i}\right)$. This modeling yields the probability distribution of $a / R_{\star}$ via a Monte Carlo approach, which is fully described in Section 3.3. We then used the $a / R_{\star}$ distribution together with Gaussian distributions for $T_{\text {eff } \star}$ and $[\mathrm{Fe} / \mathrm{H}]$, with $1 \sigma$ uncertainties as reported previously, to estimate $M_{\star}$ and $R_{\star}$ by comparison with the stellar evolution models of Yi et al. (2001). This was done for each of the 15,000 simulations. Parameter combinations corresponding to unphysical locations in the H-R diagram ( $4 \%$ of the trials) were ignored and replaced with another randomly drawn parameter set. The resulting stellar parameters and their uncertainties were determined from the a posteriori distributions obtained in this way.

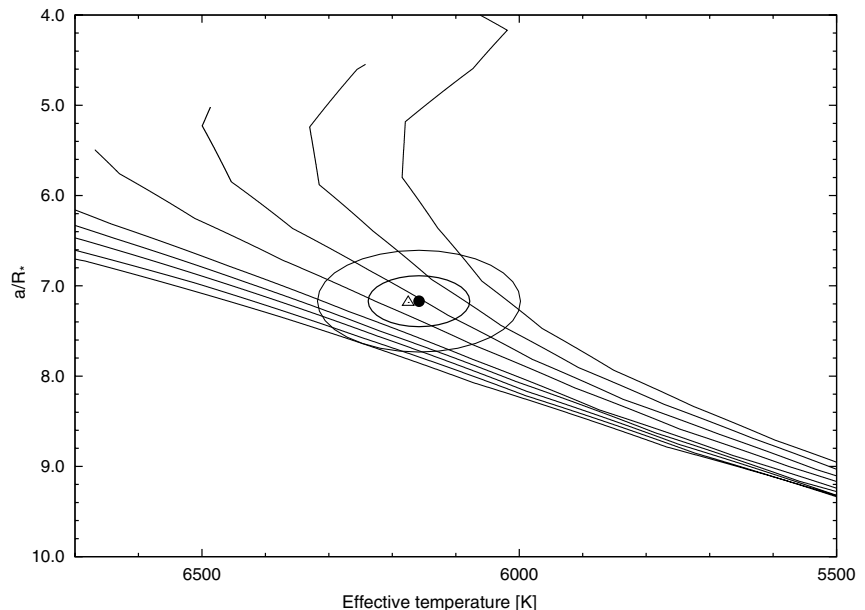

Figure 4. Stellar isochrones from Yi et al. (2001) for metallicity $[\mathrm{Fe} / \mathrm{H}]=+0.17$ and ages $0.2,0.4,0.6,0.8,1.0,1.5,2.0,2.5$, and $3.0 \mathrm{Gyr}$ (left to right). The final choice of $T_{\mathrm{eff} \star}$ and $a / R_{\star}$ is marked and encircled by the $1 \sigma$ and $2 \sigma$ confidence ellipsoids. The initial value of $T_{\mathrm{eff} \star}$ and $a / R_{\star}$ from the first SME analysis and subsequent light curve analysis is marked with a triangle that is barely offset from the final value (indicated by a filled circle).

In particular, the resulting surface gravity of $\log g_{\star}=4.34 \pm$ 0.03 is somewhat different from that derived in the initial SME analysis, which is not surprising in view of the possible strong correlations among $T_{\mathrm{eff} \star},[\mathrm{Fe} / \mathrm{H}]$, and the surface gravity. Therefore, in a second iteration, we adopted this value of $\log g_{\star}$ and held it fixed in a new SME analysis, adjusting only $T_{\text {eff } \star},[\mathrm{Fe} / \mathrm{H}]$, and $v \sin i$. This gave $T_{\text {eff }}=6158 \pm 80 \mathrm{~K}$, $[\mathrm{Fe} / \mathrm{H}]=+0.17 \pm 0.08$, and $v \sin i=3.5 \pm 0.5 \mathrm{~km} \mathrm{~s}^{-1}$, which we adopt as the final atmospheric values for the star. Finally, we repeated the calculation of the stellar mass and radius with these values and the stellar evolution models and obtained $M_{\star}=1.218 \pm 0.039 M_{\odot}, R_{\star}=1.237 \pm 0.054 R_{\odot}$, and $L_{\star}=1.97 \pm 0.22 L_{\odot}$, along with other parameters summarized in Table 3.

The model isochrones from Yi et al. (2001) for metallicity $[\mathrm{Fe} / \mathrm{H}]=+0.17$ are plotted in Figure 4 , with the final choice of effective temperature $T_{\text {eff } \star}$ and $a / R_{\star}$ marked, and encircled by the $1 \sigma$ and $2 \sigma$ confidence ellipsoids.

The stellar evolution modeling provides color indices that may be compared against the measured values as a sanity check. The best available measurements are the near-infrared magnitudes from the Two Micron All Sky Survey (2MASS) catalog (Skrutskie et al. 2006), $J_{2 \text { MASS }}=9.850 \pm 0.022$, $H_{2 \mathrm{MASS}}=9.623 \pm 0.022$, and $K_{2 \mathrm{MASS}}=9.553 \pm 0.020$, which we have converted to the photometric system of the models (ESO) using the transformations by Carpenter (2001). The resulting measured color index is $J-K=0.319 \pm 0.032$. This is within $1 \sigma$ of the predicted value from the isochrones of $J-K=0.33 \pm 0.02$. The distance to the object may be computed from the absolute $K$ magnitude from the models $\left(M_{K}=2.74 \pm 0.10\right)$ and the 2MASS $K_{s}$ magnitude, which has the advantage of being less affected by extinction than optical magnitudes. The result is $235 \pm 10 \mathrm{pc}$, where the uncertainty excludes possible systematics in the model isochrones that are difficult to quantify.

\subsection{Excluding Blend Scenarios}

We performed a line bisector analysis of the observed spectra following Torres et al. (2007) to explore the possibility that the main cause of the RV variations is actually distortions in the 
spectral line profiles due to stellar activity or a nearby unresolved eclipsing binary. The bisector analysis was performed on the Keck spectra as described in Section 5 of Bakos et al. (2007a); the spectra from the NOT were analyzed in a similar manner.

The resulting BS variations are plotted in Figure 2 and have a low amplitude compared to the orbital semi-amplitude and show no correlation with the RVs. We have set the mean of the bisector variations from the three sets of observations to zero for a better comparison between the different instruments. We conclude that there is no significant bisector variation and therefore modeling the RV variation as a result of the reflex motion of the host star of a close-in giant planet is justified.

\subsection{Global Modeling of the Data}

Our model for the follow-up light curves used analytic formulae based on Mandel \& Agol (2002) for the eclipse of a star by a planet, where the stellar flux is described by quadratic limb darkening. The limb-darkening coefficients are based on the SME results (Section 3.1) and the tables provided by Claret (2004) for the $i$ band. The transit shape was parameterized by the normalized planetary radius $R_{p} / R_{\star}$, the square of the impact parameter $b^{2}$, and the reciprocal of the half mid-ingress to midegress duration of the transit $\zeta / R_{\star}$. We chose these parameters because of their simple geometric meanings and the fact that they show negligible correlations (see Bakos et al. 2010). Our model for the HATNet data was the simplified "P1P3" version of the Mandel \& Agol (2002) analytic functions (an expansion by Legendre polynomials), for the reasons described in Bakos et al. (2010). Following the formalism presented by Pál (2009), the RV curve was modeled as an eccentric Keplerian orbit with semi-amplitude $K$ and Lagrangian orbital elements $(k, h)=e \times(\cos \omega, \sin \omega)$.

We assumed that there is a strict periodicity in the individual transit times. In practice, we assigned the transit number $N_{\text {tr }}=0$ to the first high quality follow-up light curve gathered on 2009 September 10. The adjusted parameters in the fit were the first transit center observed with HATNet, $T_{c,-284}$, and the last high quality transit center observed with the FLWO $1.2 \mathrm{~m}$ telescope, $T_{c,+18}$. The transit center times for the intermediate transits were interpolated using these two epochs and the $N_{\text {tr }}$ transit number of the actual event (Bakos et al. 2007b). The model for the $\mathrm{RV}$ data contains the ephemeris information through the $T_{c,-284}$ and $T_{c,+18}$ variables. Altogether, the eight parameters describing the physical model were $T_{c,-284}, T_{c,+18}, R_{p} / R_{\star}, b^{2}, \zeta / R_{\star}, K$, $k=e \cos \omega$, and $h=e \sin \omega$. Nine additional parameters were related to the instrumental configuration. These are the three instrumental blend factors $B_{\text {inst,i }}$ of HATNet (one for each of the three fields), which account for possible dilution of the transit in the HATNet light curve due to the wide $\left(20^{\prime \prime}\right.$ wide FWHM) point-spread function and possible crowding, the three HATNet out-of-transit magnitudes, $M_{0, \mathrm{HN}, \mathrm{i}}$, and three relative RV zeropoints $\gamma_{\text {rel,j }}$ (one each for the Keck, high-resolution FIES, and medium-resolution FIES observations).

We extended our physical model with an instrumental model that describes the systematic variations of the data. This was done in a similar fashion to the analysis presented in Bakos et al. (2010). The HATNet photometry has already been EPDand TFA-corrected before the global modeling, so we only considered systematic correction of the follow-up light curves. We chose the "ELTG" method, i.e., EPD was performed in "local" mode with EPD coefficients defined for each night, and TFA was performed in "global" mode using the same set of stars and TFA coefficients for all nights. The underlying physical
Table 4

Orbital and Planetary Parameters

\begin{tabular}{|c|c|}
\hline Parameter & Value \\
\hline \multicolumn{2}{|l|}{ Light curve parameters } \\
\hline$P$ (days) $\ldots \ldots \ldots$ & $2.775960 \pm 0.000003$ \\
\hline$T_{c}(\mathrm{BJD}) \ldots \ldots \ldots$ & $2455027.59293 \pm 0.00031$ \\
\hline$T_{14}(\text { days })^{\mathrm{a}} \ldots \ldots \ldots$ & $0.1276 \pm 0.0013$ \\
\hline$T_{12}=T_{34}(\text { days })^{\mathrm{a}} \ldots$ & $0.0150 \pm 0.0014$ \\
\hline$a / R_{\star} \ldots \ldots \ldots$ & $7.17 \pm 0.28$ \\
\hline$\zeta / R_{\star} \ldots \ldots \ldots$ & $17.73 \pm 0.10$ \\
\hline$R_{p} / R_{\star} \ldots \ldots \ldots$ & $0.1071 \pm 0.0014$ \\
\hline$b^{2} \ldots$ & $0.193_{-0.069}^{+0.063}$ \\
\hline$b \equiv a \cos i / R_{\star} \ldots \ldots \ldots$ & $0.439_{-0.098}^{+0.065}$ \\
\hline$i(\operatorname{deg}) \ldots \ldots \ldots$ & $86.6 \pm 0.7$ \\
\hline \multicolumn{2}{|l|}{ RV parameters } \\
\hline$K\left(\mathrm{~m} \mathrm{~s}^{-1}\right) \ldots \ldots \ldots$ & $531.1 \pm 2.8$ \\
\hline$k_{\mathrm{RV}}^{\mathrm{b}} \ldots \ldots \ldots$ & $-0.030 \pm 0.003$ \\
\hline$h_{\mathrm{RV}}{ }^{\mathrm{b}} \ldots \ldots \ldots$ & $-0.021 \pm 0.006$ \\
\hline$e \ldots \ldots \ldots$ & $0.036 \pm 0.004$ \\
\hline$\omega \ldots \ldots \ldots$ & $214 \pm 8^{\circ}$ \\
\hline RV jitter $\left(\mathrm{m} \mathrm{s}^{-1}\right) \ldots \ldots \ldots$ & 8.0 \\
\hline $\mathrm{RV}$ rms from fit $\left(\mathrm{m} \mathrm{s}^{-1}\right) \ldots \ldots$ & 10.0 \\
\hline \multicolumn{2}{|l|}{ Secondary eclipse parameters } \\
\hline$T_{s}(\mathrm{BJD}) \ldots \ldots \ldots$ & $2455028.929 \pm 0.005$ \\
\hline$T_{s, 14} \ldots \ldots \ldots$ & $0.1234 \pm 0.0020$ \\
\hline$T_{s, 12 \ldots \ldots \ldots}$ & $0.0142 \pm 0.0013$ \\
\hline \multicolumn{2}{|l|}{ Planetary parameters } \\
\hline$M_{p}\left(M_{\mathrm{J}}\right) \ldots \ldots \ldots$ & $4.193 \pm 0.094$ \\
\hline$R_{p}\left(R_{\mathrm{J}}\right) \ldots \ldots \ldots$ & $1.289 \pm 0.066$ \\
\hline$C\left(M_{p}, R_{p}\right)^{\mathrm{c}} \ldots \ldots \ldots$ & 0.57 \\
\hline$\rho_{p}\left(\mathrm{~g} \mathrm{~cm}^{-3}\right) \ldots \ldots \ldots$ & $2.42 \pm 0.35$ \\
\hline$a(\mathrm{AU}) \ldots \ldots \ldots$ & $0.0413 \pm 0.0004$ \\
\hline $\log g_{p}(\mathrm{cgs}) \ldots \ldots \ldots$ & $3.80 \pm 0.04$ \\
\hline$T_{\mathrm{eq}}(\mathrm{K}) \ldots \ldots \ldots$ & $1626 \pm 40$ \\
\hline$\Theta^{\mathrm{d}} \ldots \ldots \ldots$ & $0.220 \pm 0.011$ \\
\hline$\langle F\rangle\left(10^{9} \mathrm{erg} \mathrm{s}^{-1} \mathrm{~cm}^{-2}\right)^{\mathrm{e}} \ldots \ldots$ & $1.58 \pm 0.16$ \\
\hline
\end{tabular}

Notes.

${ }^{a} T_{14}$ : total transit duration, time between first to last contact; $T_{12}=T_{34}$ : ingress/egress time, time between first and second or third and fourth contact.

b Lagrangian orbital parameters derived from the global modeling and primarily determined by the RV data.

c Correlation coefficient between the planetary mass $M_{p}$ and radius $R_{p}$.

d The Safronov number is given by $\Theta=\frac{1}{2}\left(V_{\text {esc }} / V_{\text {orb }}\right)^{2}=$ $\left(a / R_{p}\right)\left(M_{p} / M_{\star}\right)$ (see Hansen \& Barman 2007).

e Incoming flux per unit surface area.

model was based on the Mandel \& Agol (2002) analytic formulae, as described earlier. The five EPD parameters were the hour angle (characterizing a monotonic trend that linearly changes over time), the square of the hour angle, and the stellar profile parameters (equivalent to FWHM, elongation, and position angle). The functional form of the above parameters contained six coefficients, including the auxiliary out-of-transit magnitude of the individual events. The EPD parameters were independent for all four nights, implying 24 additional coefficients in the global fit. For the global TFA analysis, we chose 20 template stars that had good quality measurements for all nights and on all frames, implying an additional 20 parameters in the fit. Thus, the total number of fitted parameters was 17 (physical model) + $24($ local EPD $)+20$ (global TFA $)=61$.

The joint fit was performed as described in Bakos et al. (2010). We minimized the $\chi^{2}$ in the parameter space by using a hybrid 


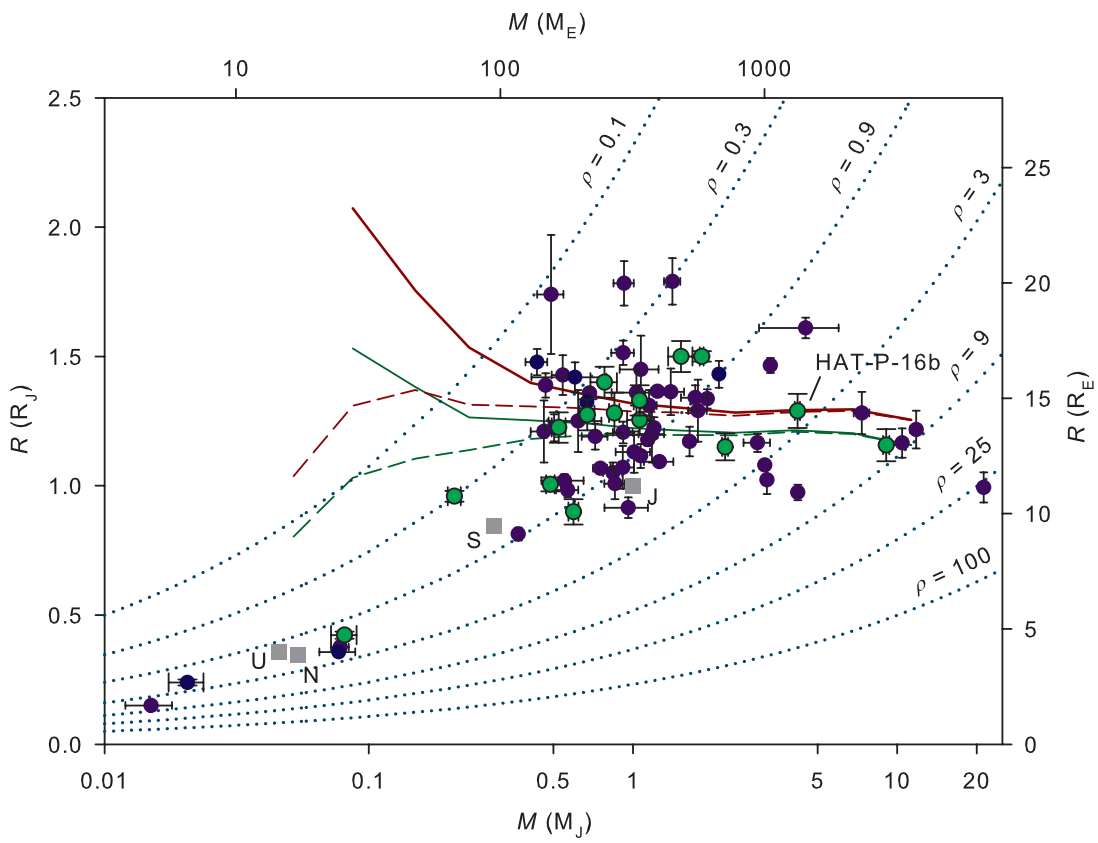

Figure 5. Mass-radius diagram of currently known TEPs. HATNet planets are show in green and HAT-P-16b is labeled explicitly. The solar system planets are shown as filled gray squares. Isodensity curves (in $\mathrm{g} \mathrm{cm}^{-3}$ ) are plotted as dotted lines. Overlaid are planetary $1.0 \mathrm{Gyr}$ (brown, the upper set of lines) and $4.5 \mathrm{Gyr}$ (green, the lower set of lines) isochrones from Fortney et al. (2007) for $\mathrm{H} /$ He-dominated planets with core masses of $M_{c}=0 M_{\oplus}$ (solid) and $M_{c}=10 M_{\oplus}$ (dashed), interpolated to the solar-equivalent semimajor axis of HAT-P-16b.

(A color version of this figure is available in the online journal.)

algorithm, combining the downhill simplex method (AMOEBA; see Press et al. 1992) with the classical linear least-squares algorithm. Uncertainties for the parameters were derived using the Markov Chain Monte Carlo method (see Ford 2006; Pál 2009).

The eccentricity of the system appeared as significantly non zero $(k=-0.030 \pm 0.003, h=-0.021 \pm 0.006)$. The best-fit results for the relevant physical parameters are summarized in Table 4. We also list the RV "jitter," which is a quantity added in quadrature to the calculated RV measurement uncertainties in order to have $\chi^{2} /$ dof $=1$ from the RV data for the global fit. The "jitter" may be of astrophysical origin (pulsations, activity) or it may be in part due to instrumental effects.

In addition, some auxiliary parameters (not listed in the table) are: $T_{\mathrm{c},-284}=2454297.5154 \pm 0.0009(\mathrm{BJD}), T_{\mathrm{c},+18}=$ $2455135.8554 \pm 0.0003$ (BJD), $\gamma_{\text {rel }}=4.5 \pm 3.8 \mathrm{~m} \mathrm{~s}^{-1}$, $\gamma_{\text {rel,FIEShi }}=-434.9 \pm 4.2 \mathrm{~m} \mathrm{~s}^{-1}$ (FIES, high resolution), $\gamma_{\text {rel,FIESmed }}=-442.9 \pm 2.8 \mathrm{~m} \mathrm{~s}^{-1}$ (FIES, medium resolution), $B_{\text {instr, } 123}=0.77 \pm 0.08, B_{\text {instr, } 163}=0.93 \pm 0.04$, and $B_{\text {instr }, 164}=$ $0.96 \pm 0.04$. Note that these gamma velocities do not correspond to the true center-of-mass RV of the system, but are only relative offsets. The true systemic velocity of the system is RV = $-16.83 \pm 0.19 \mathrm{~km} \mathrm{~s}^{-1}$, as described in Section 2.2. The planetary parameters and their uncertainties can be derived by the direct combination of the a posteriori distributions of the light curve, RV, and stellar parameters. We found that the planet is fairly massive with $M_{p}=4.193 \pm 0.094 M_{\mathrm{J}}$, and compact with radius of $R_{p}=1.289 \pm 0.066 R_{\mathrm{J}}$, yielding a mean density of $\rho_{p}=2.42 \pm 0.35 \mathrm{~g} \mathrm{~cm}^{-3}$. The final planetary parameters are summarized at the bottom of Table 4 .

\section{DISCUSSION}

We present the discovery of HAT-P-16b with a period of $P=2.7760$ days, a mass of $M_{p}=4.19 \pm 0.09 M_{\mathrm{J}}$, and a radius of $R_{p}=1.29 \pm 0.07 R_{\mathrm{J}}$. Currently, there are only a handful of planets residing near HAT-P-16b in the mass-radius diagram. CoRoT-2b (Alonso et al. 2008) and CoRoT-6b (Fridlund et al. 2010) have similar masses and periods, but both have eccentricities consistent with zero. HD 80606 (Naef et al. 2001) and HD 17156 (Fischer et al. 2007) are also similar in mass, but both planets have long periods and very eccentric orbits. WASP-10b ( $P=3.09$ days, $e=0.06, M_{p}=2.96 M_{\mathrm{J}}$, and $R_{p}=1.08 R_{\mathrm{J}}$; see Christian et al. 2009; Johnson et al. 2009) is probably the transiting planet which most resembles HAT-P-16b with a similar period and eccentricity, albeit with a slightly lower mass and radius. At $V=10.8$ mag, HAT-P-16 is one of the brightest stars known to host a $\sim 4 M_{\text {J }}$ planet.

We have compared HAT-P-16b to the theoretical models from Fortney et al. (2007) by interpolating the models to the solarequivalent semimajor axis of $a_{\text {equiv }}=0.0294 \pm 0.0014 \mathrm{AU}$, the result of which can be seen overplotted in Figure 5. We find that the mass and radius of HAT-P-16b are quite consistent with the $1 \mathrm{Gyr}$ model and conclude that HAT-P-16b is most likely an $\mathrm{H} / \mathrm{He}$-dominated planet.

The RV data set used to characterize HAT-P-16b consists of three different velocity sets from two different telescopes (FIES medium- and high-resolution spectra from the NOT and HIRES spectra from Keck I), requiring additional free parameters in the global fit to account for the arbitrary velocity offset between the measurements. Nevertheless, the different observations yield very similar semi-amplitude and we find an rms from the bestfit model of only $10.0 \mathrm{~m} \mathrm{~s}^{-1}$. The rich data set consisting of $27 \mathrm{RV}$ observations allows us to accurately determine the small non-zero eccentricity of the orbit as $e=0.036$ with a high significance of $10 \sigma$, with $k \neq 0$ at $10 \sigma$ and $h \neq 0$ at $3 \sigma$. The eccentricity is consistent and clearly evident even when fitting separate orbits to the three different velocity data sets. 
Most short-period TEPs have eccentricities consistent with zero, as is expected because of circularization driven by tidal dissipation within the planet with a typical timescale substantially less than $1 \mathrm{Gyr}$ (see Mardling 2007). In fact, the estimated circularization timescale for HAT-P-16b is $\tau_{\text {cir }} \sim 0.03 \mathrm{Gyr}$ (when the tidal dissipation parameter is assumed to be $Q_{p}=10^{5}$ ), which is much less than the estimated $\sim 2$ Gyr age of the system. On the other hand, Matsumura et al. (2008) and Jackson et al. (2008) argue that circularization timescales could be significantly higher and the planets with eccentric orbits may simply be in the process of being circularized. Adams \& Laughlin (2006) argue that for multiple systems with a hot Jupiter as the inner planet, secular excitation of the eccentricity by companion planets could explain the non-zero eccentricity of these systems. Thus, the origin of the eccentricity of HAT-P-16b remains unclear.

With a stellar rotation of $3.5 \pm 0.5 \mathrm{~km} \mathrm{~s}^{-1}$ and an impact parameter of $b \sim 0.43$, HAT-P-16 is a favorable target for measuring the Rossiter-McLaughlin (RM) effect. This effect can be used to determine the alignment of the planet's orbital angular momentum vector with the stellar spin axis (Winn et al. 2009). The estimated amplitude of the RM effect is $47 \mathrm{~m} \mathrm{~s}^{-1}$ for HAT-P-16b.

HATNet operations have been funded by NASA grants NNG04GN74G, NNX08AF23G, and SAO IR\&D grants. The work of G.Á.B. and J.A.J. were supported by the Postdoctoral Fellowship of the NSF Astronomy and Astrophysics Program (AST-0702843 and AST-0702821, respectively). G.T. acknowledges partial support from NASA grant NNX09AF59G. We acknowledge partial support also from the Kepler Mission under NASA Cooperative Agreement NCC2-1390 (D.W.L., PI). G.K. thanks the Hungarian Scientific Research Foundation (OTKA) for support through grant K-81373. Based in part on observations made with the Nordic Optical Telescope, operated on the island of La Palma jointly by Denmark, Finland, Iceland, Norway, and Sweden, in the Spanish Observatorio del Roque de los Muchachos of the Instituto de Astrofisica de Canarias. This research has made use of Keck telescope time granted through NASA (N018Hr).

\section{REFERENCES}

Adams, F. C., \& Laughlin, G. 2006, ApJ, 649, 992

Alonso, R., et al. 2004, ApJ, 613, L153

Alonso, R., et al. 2008, A\&A, 482, L21

Auvergne, M., et al. 2009, A\&A, 506, 411
Bakos, G. Á., Noyes, R. W., Kovács, G., Stanek, K. Z., Sasselov, D. D., \& Domsa, I. 2004, PASP, 116, 266

Bakos, G. Á., et al. 2007a, ApJ, 670, 826

Bakos, G. Á., et al. 2007b, ApJ, 670, 826

Bakos, G. Á., et al. 2010, ApJ, 710, 1724

Borucki, W. J., et al. 2010, Science, 327, 977

Butler, R. P., Marcy, G. W., Williams, E., McCarthy, C., Dosanjh, P., \& Vogt, S. S. 1996, PASP, 108, 500

Carpenter, J. M. 2001, AJ, 121, 2851

Christian, D. J., et al. 2009, MNRAS, 392, 1585

Claret, A. 2004, A\&A, 428, 1001

Djupvik, A. A., \& Andersen, J. 2009, arXiv:0901.4015

Droege, T. F., Richmond, M. W., \& Sallman, M. 2006, PASP, 118, 1666

Fischer, D. A., et al. 2007, ApJ, 669, 1336

Ford, E. 2006, ApJ, 642, 505

Fortney, J. J., Marley, M. S., \& Barnes, J. W. 2007, ApJ, 659, 1661

Fridlund, M., et al. 2010, A\&A, 512, A14

Hansen, B. M. S., \& Barman, T. 2007, ApJ, 671, 861

Hewett, P. C., Irwin, M. J., Bunclark, P., Bridgeland, M. T., Kibblewhite, E. J., He, X. T., \& Smith, M. G. 1985, MNRAS, 213, 971

Horne, K. 1986, PASP, 98, 609

Jackson, B., Greenberg, R., \& Barnes, R. 2008, ApJ, 678, 1396

Johnson, J. A., Winn, J. N., Cabrera, N. E., \& Carter, J. A. 2009, ApJ, 692, L100

Kovács, G., Bakos, G. Á., \& Noyes, R. W. 2005, MNRAS, 356, 557

Kovács, G., Zucker, S., \& Mazeh, T. 2002, A\&A, 391, 369

Latham, D. W. 1992, in ASP Conf. Ser. 32, Complementary Approaches to Double and Multiple Star Research, ed. H. A. McAlister \& W. I. Hartkopf (San Francisco: ASP), 110

Latham, D. W., et al. 2010, ApJ, 713, L140

Mandel, K., \& Agol, E. 2002, ApJ, 580, L171

Marcy, G. W., \& Butler, R. P. 1992, PASP, 104, 270

Mardling, R. A. 2007, MNRAS, 382, 1768

Matsumura, S., Takeda, G., \& Rasio, F. A. 2008, ApJ, 686, L29

McCullough, P. R., Stys, J. E., Valenti, J. A., Fleming, S. W., Janes, K. A., \& Heasley, J. N. 2005, PASP, 117, 783

Naef, D., et al. 2001, A\&A, 375, 27

Noyes, R. W., Hartmann, L. W., Baliunas, S. L., Duncan, D. K., \& Vaughan, A. H. 1984, ApJ, 279, 763

Pál, A. 2009, MNRAS, 396, 1737

Pollacco, D. L., et al. 2006, PASP, 118, 1407

Press, W. H., Teukolsky, S. A., Vetterling, W. T., \& Flannery, B. P. 1992 , Numerical Recipes in C: The Art of Scientific Computing (2nd ed.; Cambridge: Cambridge Univ. Press)

Skrutskie, M. F., et al. 2006, AJ, 131, 1163

Sozzetti, A., Torres, G., Charbonneau, D., Latham, D. W., Holman, M. J., Winn, J. N., Laird, J. B., \& O'Donovan, F. T. 2007, ApJ, 664, 1190

Torres, G., Neuhäuser, R., \& Guenther, E. W. 2002, AJ, 123, 1701

Torres, G., et al. 2007, ApJ, 666, 121

Torres, G., et al. 2010, ApJ, 715, 458

Valenti, J. A., \& Fischer, D. A. 2005, ApJS, 159, 141

Valenti, J. A., \& Piskunov, N. 1996, A\&AS, 118, 595

Vaughan, A. H., Preston, G. W., \& Wilson, O. C. 1978, PASP, 90, 267

Vogt, S. S., et al. 1994, Proc. SPIE, 2198, 362

Winn, J. N., Johnson, J. A., Albrecht, S., Howard, A. W., Marcy, G. W., Crossfield, I. J., \& Holman, M. J. 2009, ApJ, 703, L99

Yi, S. K., Demarque, P., Kim, Y.-C., Lee, Y.-W., Ree, C. H., Lejeune, T., \& Barnes, S. 2001, ApJS, 136, 417 